\title{
Nailfold capillary microscopy for evaluating hand-arm vibration syndrome
}

\author{
Chan Boo Lee ${ }^{1}$, Joo Hyun Sung ${ }^{1}$, Jung Hun Park', Cheol In Yoo', Chang Sun Sim¹, Ji Seon Oh² and Hun Lee
}

\begin{abstract}
Objectives: We evaluated nailfold capillary abnormalities in patients with hand-arm vibration syndrome using nailfold capillary microscopy.

Methods: Fifty workers who underwent a special health examination because of exposure to hand-arm vibration at Ulsan University Hospital in 2012 (exposed group) and a control group of 50 white-collar employees were evaluated through a questionnaire survey regarding their present tasks, types of tools used, vibration exposure duration, use of protective wear, and medical history. Then, an occupational physician performed a physical examination for any hand deformities, skin problems, or motor and sensory dysfunctions of the upper extremities. The nailfold capillary morphologies (tortuous, crossing, bushy, meandering, branching, hemorrhage, avascular area, enlarged, and giant), capillary dimensions (afferent, top, venous, total width, and length), and specific counts (crossing and branching) on both fourth fingers were determined by a rheumatologist. Thereafter, the exposed subjects were assessed according to the Stockholm workshop classification scale. In total, 8 and 6 subjects in the exposed and control groups, respectively, were excluded from the study because of poor capillary microscopic image quality. In addition, 24 subjects in the exposed group with Stockholm vascular stage 0 were excluded. Finally, capillary morphology, dimensions, and specific counting were compared between the exposed $(n=18)$ and control groups $(n=44)$.

Results: The exposed group had significantly greater crossing capillaries and abnormal capillary numbers that included crossing capillaries (crossing, branching, bushy, and meandering) but smaller branching and abnormal capillary numbers that excluded crossing capillaries (branching, bushy, and meandering) than the control group did. No significant difference in capillary dimensions was observed between the two groups. Despite the adjustment for age, smoking status, and underlying diseases, the statistical significance was unchanged. In the specific counting of the type of capillaries, the exposed group had a significantly higher total crossing count but fewer total branching count than the control group did. However, no statistical significance resulted after adjustment for age, smoking status, and underlying diseases.
\end{abstract}

Conclusions: In this study, the exposed group had significantly more crossing capillaries and a higher crossing count than the control group did.

Keywords: Hand-arm vibration syndrome, Nailfold capillary microscopy, Crossing capillary number, Crossing count

\section{Introduction}

Hand-arm vibration is defined as the transmission of vibrations produced by handheld tools to the hands and arms of the tool-wielding individual. Hand-arm vibration syndrome (HAVS) is the manifestation of one or more specific neurological, vascular, and musculoskeletal disorders that are causally associated with vibration exposures

\footnotetext{
* Correspondence: leeheun67@gmail.com

'Department of Occupational and Environmental Medicine, Ulsan University Hospital, University of Ulsan, College of Medicine, Ulsan, Korea

Full list of author information is available at the end of the article
}

from handheld vibrating tools. In particular, the syndrome is known to be a complex combination of disorders that insidiously and passively affect HAVS patients [1]. In England, approximately 288,000 people have HAVS. According to the Medical Research Council Survey conducted between 1997 and 1998, nearly 5 million people are exposed to hand-transmitted vibrations at work in a given week, most of whom are male, with a male-to-female ratio of approximately 8:1 among those who have developed HAVS [2]. Most of the studies associated with HAVS have

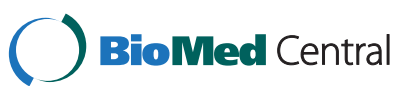

(c) 2014 Lee et al.; licensee BioMed Central. This is an Open Access article distributed under the terms of the Creative Commons Attribution License (http://creativecommons.org/licenses/by/4.0), which permits unrestricted use, distribution, and reproduction in any medium, provided the original work is properly credited. The Creative Commons Public Domain Dedication waiver (http://creativecommons.org/publicdomain/zero/1.0/) applies to the data made available in this article unless otherwise stated. 
shown a positive correlation between exposure to high levels of hand-transmitted vibrations and the presence of vascular symptoms of HAVS [2]. The risk for HAVS was shown to be highest in occupations that use pneumatic drills, grinders, and impact wrenches [3]. Moreover, the incidence of HAVS was approximately 70\% among these high-risk individuals [3].

Various methods to measure and evaluate the status of vascular disorders have been used, including the measurement of the recovery time after nail compression, nailfold capillary microscopy, plethysmography, thermometry and thermography, and laser Doppler imaging and flowmetry. These tests are used to evaluate skin microcirculation and morphology of capillary. Among these tests, nailfold capillary microscopy is considered one of the established methods to diagnose and monitor collagen-vascular diseases because it can evaluate structural changes in the superficial cutaneous vasculature $[4,5]$.

Capillary microscopy is performed most commonly to identify patients with Raynaud's phenomenon. Through this method, patients with scleroderma (systemic sclerosis) who exhibit a high prevalence of Raynaud's phenomenon can be easily differentiated from patients with primary Raynaud's phenomenon or Raynaud's phenomenon caused by other connective tissue diseases because most patients with scleroderma (systemic sclerosis) exhibit pathognomonic changes under capillary microscopy [6,7]. Similarly, capillary microscopy can also be applied to HAVS which is a type of secondary Raynaud's phenomenon.

Outside of South Korean research on HAVS diagnosis using capillary microscopy, Kaji et al. [8] retrospectively analyzed 98 HAVS patients among 294 subjects who were exposed to hand-transmitted vibrations. In their study, the capillary morphology was differentiated into three stages (stages A, B, and C) according to the Fagrell and Lundberg classification, and then the results were compared with the control group. Among the HAVS patients in their study, stage B classification (indistinct capillaries) was the most common diagnosis accounting for $42.9 \%$ of the patients. In the control group, stage A classification (dot- or comma-shaped appearance with good tonicity) was the most common diagnosis accounting for $50.0 \%$ of the subjects in the control group. However, because the capillary microscopy results of both groups were differentiated into three stages simply based on the distinctiveness of their capillary morphology, the lack of a more specific parameter was a limitation of their study. Moreover, Littleford et al. [9] compared the capillary morphology between 10 HAVS patients and 10 control group subjects. Interestingly, their study implemented a scoring system for certain measured parameters including capillary dropout, tortuosity, subpapillary venular plexus visualization, and disarrangement of capillary polarity. However, the capillary morphologies of the control group were all found to be normal; therefore, comparison with the patient group was difficult. Moreover, their study had a relatively small study population that further limited their findings.

The diagnostic method that measures recovery time after nail compression to confirm the presence of vascular disorders in HAVS patients can yield different applied pressures and recovery times depending on the skills and experience of the examiner. The reproducibility of this diagnostic method is also unknown, thus it is not preferred [4]. Furthermore, thermometry and thermography tends to exhibit inconsistent sensitivity and specificity depending on the study [4]. In addition, a limitation of laser Doppler flowmetry is that it has low reproducibility in microcirculation studies based on physiological changes [4]. Plethysmography is expensive, complicated, and requires a skilled examiner to ensure the accuracy of the test results [4]. Owing to the problems posed by the aforementioned diagnostic methods, cold provocation test and finger angiography are commonly used. However, even these alternative methods have limitations. In particular, the cold provocation test is time intensive and subject to the influence of seasonal changes on skin temperature [10,11]. Moreover, finger angiography is invasive. However, nailfold capillary microscopy is a simple, noninvasive procedure that can quickly evaluate the status of capillary disorders. Nonetheless, the use of capillary microscopy to diagnose HAVS in South Korean patients has not been well studied. Therefore, the present study is a pilot study that attempts to test a new diagnostic approach to evaluating HAVS in South Korea.

\section{Materials and methods}

\section{Study participants}

In 2012, 50 subjects who visited Ulsan University Hospital for a special health examination after being exposed to hand-arm vibrations presented with paleness, tingling sensations, or numbness of the hands. All subjects underwent nailfold capillary microscopy. The images of eight subjects were low quality (based on whether both sides and the center of the capillary were visible) and 24 subjects were found to have a Stockholm vascular stage of zero in the cold provocation test; thus, 32 subjects were excluded. The remaining 18 subjects were selected as the exposed group. The control group was selected from a group of 50 white-collar employees who had never been exposed to localized vibrations. Of these, six subjects were excluded because their nailfold capillary microscopic images were of poor quality; therefore, a total of 44 workers were included in the control group.

\section{Survey and physical examination}

Variables associated with vibrations that were investigated included age, present task, type of vibrating tool used, the 
duration of use, occupational posture, safety equipment status, and method of commute to their workplace. Furthermore, data on subject's medical history (hypertension, heart disease, diabetes mellitus, musculoskeletal disorders, trauma, etc.), alcohol consumption, and smoking history were collected because these variables may influence any current symptoms. In the physical examination, an occupational and environmental medicine specialist evaluated all subjects for nail changes, finger deformities, skin disorders of the hands, musculoskeletal disorders of the entire upper limb, tendon reflex disorder, upper extremity range of motion, and the level of pain during these motions.

\section{Nailfold capillary microscopy and Stockholm staging}

Prior to Stockholm staging, nailfold capillary microscopy was performed to prevent any adverse effects such as vasoconstriction due to the cold water used in the cold provocation test. The capillary microscope was a CM1000 (CM, 2009, Seoul, South Korea), and the software i-Solution/Lite (iMT i-Solution Inc.) was used to analyze the images.

Capillary microscopy was performed after the subjects rested for 15 to 30 minutes at a standardized room temperature $\left(20-23^{\circ} \mathrm{C}\right)$. The nailfolds of the fourth fingers of both hands were examined because this finger provides superior capillary visibility over all the fingers $[12,13]$. Two sections of the fourth finger on each hand were marked and spanned $5 \mathrm{~mm}$. Then, immersion oil or liquid paraffin was applied onto the skin to maximize the transparency of the keratin layer of the epithelial cells [14]. All subjects underwent high- $(\times 200)$ and low-power $(\times 100)$ microscopic examinations, with the total examination time ranging from 15 to 30 minutes. However, the dimensions of all the capillaries (afferent, top, venous, total width, and length) were not measured. Thus, the three largest or most abnormal capillaries were selected and examined. In the case of the morphological parameters (tortuous, crossing, bushy, meandering, branching, hemorrhagic, avascular area, enlarged, and giant), the number of capillaries that displayed these parameters within a 2-mm area was counted. In the case of tortuous capillaries, a more complex definition of the morphology was used for any crossing, meandering, or branching capillaries. The capillary morphologies were defined as follows: crossing, containing at least 1 circle in the capillary loop; branching, having at least 1 loop bulging with the exception of the capillary top; bushy, having a more complex morphology than branching; giant, having a capillary top larger than $50 \mu \mathrm{m}$; enlarged, surpassing the average capillary top size of $15 \mu \mathrm{m}$; and avascular area, the absence of capillaries within twice the shortest distance between the nailfold capillaries as observed by the examiner. Furthermore, a combined count of the crossing, meandering, branching, and bushy capillary counts was computed. However, the counts of four particular capillaries were not combined for a specific reason. Under the high- $(\times 200)$ and low-power $(\times 100)$ microscopic examinations, the number of hairpinshaped capillaries within a $1-\mathrm{mm}$ area was counted to compute their respective densities, and the lowest value of the measurable range was used. The average density was defined as the average between the high- and low-power densities. In addition, the number of crossing and branching abnormalities was counted by selecting three capillaries that reflected the most severe crossing and branching ones, respectively, based on the capillary microscopic findings. As standard measures of severity, the number of circles was used as the crossing count and the number of capillaries bulging outside aside from the capillary top was used as the branching count.

\section{Statistical analysis}

A $t$ test was performed to compare the mean age between the exposed and control group. Chi-square tests were used to compare the smoking statuses and medical histories between the two groups. In addition, a $t$ test was used to compare the morphology and dimensions (descriptive factors) of the capillary microscopic findings between the two groups. Moreover, analysis of covariance was used to determine the statistical significance of the results after adjusting for age, smoking status, and underlying diseases. However, smoking status and underlying diseases were analyzed as dummy variables because they are categorical variables. Statistical analyses were performed using SPSS ver. 21 (IBM SPSS Inc., Chicago, IL), and a $p<0.05$ was considered statistically significant.

\section{Results}

\section{Characteristics of the study participants}

The mean age of the 18 exposed subjects was $57.1 \pm$ 4.9 years, whereas that of the 44 subjects in the control group was $44.5 \pm 11.0$ years. The mean vibration-exposure duration in the exposed group was $25.6 \pm 6.7$ years; the most common tools being the grinder (61.1\%) and rock drill (38.9\%). Among the tasks, painting and fitting were $27.8 \%$ and $22.2 \%$, respectively, mining was the most common, at a frequency of $33.3 \%$. Current smokers comprised the majority of the exposed and control groups at $44.4 \%$ and $38.6 \%$, respectively; however, the difference was not statistically significant. The alcohol consumption statuses in the exposed group were $57.1 \%$ and $42.9 \%$ for drinkers and non-drinkers, respectively. In addition, $72.2 \%$ of the exposed group did not have a history of disease, $11.1 \%$ had hypertension, $11.1 \%$ had other diseases, and $5.6 \%$ had finger injuries. In the control group, $93.8 \%$ did not have any history of illness and $6.3 \%$ had a history of hypertension. However, the difference between the medical histories of the two groups was not significant. Regarding the use of 
protective gloves, $66.7 \%$ of the exposed group reported wearing only cotton gloves, $22.2 \%$ reported that they always wear antivibration gloves, and $11.1 \%$ reported that they do not wear any gloves. For the mode of transportation used to commute to work in the exposed group, $46.7 \%$ rode a motorcycle, $46.7 \%$ rode a bus, and $6.7 \%$ walked to work (Table 1). Moreover, the most commonly used (at 77.8\%) occupational postures were raising the arms above the shoulders, bending the neck forward or backward, squatting, and bending the back. Other

\section{Table 1 Characteristics of the exposed subjects}

\begin{tabular}{|c|c|c|c|}
\hline & $\begin{array}{l}\text { VWF } \\
(n=18)\end{array}$ & $\begin{array}{l}\text { Non-VWF } \\
(n=44)\end{array}$ & $p$ value \\
\hline Age (years) & $57.1 \pm 4.9$ & $44.5 \pm 11.0$ & 0.001 \\
\hline Vibration exposure (years) & $25.6 \pm 6.7$ & - & \\
\hline \multicolumn{4}{|l|}{ Smoking $^{\dagger}(\%)$} \\
\hline Non-smoker & 33.3 & 29.5 & 0.751 \\
\hline Ex-smoker & 22.2 & 31.8 & \\
\hline Current smoker & 44.4 & 38.6 & \\
\hline \multicolumn{4}{|l|}{ Alcohol consumption (\%) } \\
\hline Non-drinker & 42.9 & - & \\
\hline Drinker & 57.1 & - & \\
\hline \multicolumn{4}{|l|}{ Past medical history ${ }^{\dagger}(\%)$} \\
\hline No past disease & 72.2 & 93.8 & 0.098 \\
\hline Hypertension & 11.1 & 6.3 & \\
\hline Finger injury & 5.6 & - & \\
\hline Other diseases & 11.1 & - & \\
\hline \multicolumn{4}{|l|}{ Source of vibration (\%) } \\
\hline Grinder & 61.1 & - & \\
\hline Rock drill & 38.9 & - & \\
\hline \multicolumn{4}{|l|}{ Antivibration gloves (\%) } \\
\hline Nothing & 11.1 & - & \\
\hline Only cotton gloves & 66.7 & - & \\
\hline Always & 22.2 & - & \\
\hline \multicolumn{4}{|l|}{ Tasks (\%) } \\
\hline Painting & 27.8 & - & \\
\hline Fitting & 22.2 & - & \\
\hline Welding & 11.1 & - & \\
\hline Mining & 33.3 & - & \\
\hline Digging & 5.6 & - & \\
\hline \multicolumn{4}{|c|}{ Mode of transportation to work (\%) } \\
\hline Walking & 6.7 & - & \\
\hline Motorcycle & 46.7 & - & \\
\hline Bus & 46.7 & - & \\
\hline
\end{tabular}

Mean \pm SD.

${ }^{*} t$ test.

${ }^{\dagger}$ Chi-square test.

Abbreviations: VWF vibration-induced white finger, Non-VWF non- vibrationinduced white finger. common responses included only 2 or 3 of these postures (data not shown).

\section{Capillary microscopic findings of the exposed and control groups}

The imaging results of the nailfold capillary microscopy in the exposed and control groups indicated that the numbers of crossing capillaries, avascular areas, enlarged capillaries, and abnormal capillaries (crossing, meandering, branching, and bushy) were higher in the exposed group than in the control group. However, the numbers of tortuous, bushy, meandering, branching, and abnormal capillaries (meandering, branching, bushy) and the mean density were higher in the control group than in the exposed group. The numbers of capillaries that were crossing, branching, abnormal including crossing (i.e., crossing, meandering, branching, and bushy), and abnormal excluding crossing (i.e., meandering, branching, and bushy) significantly differed between the two groups. After adjusting for age, smoking status, and underlying diseases, none of the categories was significantly different between the two groups (Table 2).

The venous capillary size and total width were smaller in the exposed group than in the control group. Afferent capillary size, top capillary size, and capillary length were greater in the exposed group than in the control group, but the results were not statistically significant. The significance of the results did not change even after adjusting for age, smoking status, and underlying diseases (Table 3).

In the specific counting of the morphological findings, the exposed group exhibited a higher total crossing count, whereas the control group exhibited a higher total branching count. While the unadjusted results were statistically significant, the fully adjusted results were not (Table 4).

\section{Discussion}

The most common cause of Raynaud's phenomenon is the exaggerated response of the blood vessels due to cold or emotional stress [15]. However, the morphological changes of the capillaries in Raynaud's phenomenon are related to capillary wall damage due to long-term exposure to vibration stimulation, not to the changes in blood flow through the autonomic nervous system. Therefore, the morphological classification of capillaries is useful as a method of differential diagnosis for HAVS [16]. However, considering the wide degree of individual variations in capillary morphology even in the control group, this method is clearly limited as a diagnostic tool.

However, since Maurice Raynaud (1834-1881) presented his theory of local ischemic damage in the hands, feet, nose, and tongue, capillary microscopy has been recognized as an important tool to visualize microvascular 
Table 2 Morphological findings of the nailfold capillary microscopy test (non-VWF vs. VWF)

\begin{tabular}{|c|c|c|c|c|c|}
\hline \multicolumn{2}{|c|}{ Microscopic findings } & \multirow{2}{*}{$\begin{array}{l}\text { Non-VWF }(\mathbf{n}=\mathbf{4 4}) \\
2.11 \pm 2.13\end{array}$} & \multirow{2}{*}{$\begin{array}{l}\text { VWF }(\mathbf{n}=\mathbf{1 8}) \\
1.94 \pm 2.34\end{array}$} & \multirow{2}{*}{$\begin{array}{l}p \text { value }^{*} \\
0.783\end{array}$} & \multirow{2}{*}{$\begin{array}{l}\text { Adjusted } \boldsymbol{p} \text { value } \\
0.464\end{array}$} \\
\hline & Tortuous & & & & \\
\hline & Crossing & $3.61 \pm 3.03$ & $5.89 \pm 3.79$ & 0.015 & 0.915 \\
\hline & Bushy & $0.14 \pm 0.90$ & $0.00 \pm 0.00$ & 0.527 & - \\
\hline & Meandering & $0.27 \pm 1.09$ & $0.11 \pm 0.32$ & 0.539 & 0.953 \\
\hline \multirow[t]{5}{*}{ Morphology } & Branching & $1.02 \pm 1.49$ & $0.28 \pm 0.75$ & 0.012 & 0.142 \\
\hline & Hemorrhage & $0.00 \pm 0.00$ & $0.00 \pm 0.00$ & - & - \\
\hline & Avascular area & $0.66 \pm 0.96$ & $0.72 \pm 0.89$ & 0.812 & 0.562 \\
\hline & Enlarged & $1.64 \pm 2.24$ & $1.89 \pm 2.05$ & 0.682 & 0.714 \\
\hline & Giant & $0.00 \pm 0.00$ & $0.00 \pm 0.00$ & - & - \\
\hline \multirow[t]{2}{*}{ Combined count } & Abnormal capillary (including crossing) & $3.80 \pm 2.96$ & $6.00 \pm 3.66$ & 0.016 & 0.933 \\
\hline & Abnormal capillary (excluding crossing) & $1.11 \pm 1.62$ & $0.33 \pm 0.77$ & 0.013 & 0.184 \\
\hline \multicolumn{2}{|c|}{ Average density (capillary number/mm) } & $6.70 \pm 0.92$ & $6.69 \pm 1.06$ & 0.970 & 0.764 \\
\hline
\end{tabular}

Unit: capillary number.

${ }^{*} t$ test.

${ }^{\dagger}$ Adjusted for age, smoking status, and diseases using analysis of covariance.

Abbreviations: Non-VWF non- vibration-induced white finger, VWF vibration-induced white finger.

dysfunction, a characteristic of Raynaud's phenomenon $[6,17]$. The peripheral microangiopathy of systemic sclerosis was also easily identifiable and studied through capillary microscopy. The capillary morphological abnormalities in systemic sclerosis can largely be categorized as either minor or major. Minor abnormalities are commonly found even in healthy individuals, consisting of tortuous, crossing, and enlarged capillaries. Major abnormalities that are rarely found in normal persons and considered pathological in nature are giant, bushy, meandering, and branching capillaries [14]. However, one major difference from the results of the present study compared to those of previous studies is that branching capillaries were significantly more common in the control group than the exposed group, and the minor nonpathological abnormality such as the crossing capillary was significantly more common in the exposed group than the control group. Nevertheless, it is unknown whether the pathological findings in systemic sclerosis are also pathological in cases of HAVS. Moreover, although it is normal for some control group subjects to exhibit abnormal findings for some parameters, our results may not be entirely able to display these findings. However, one of the possible interpretations for this difference can be that the pathophysiological mechanisms of systemic sclerosis and HAVS are fundamentally different. Although the primary cause of systemic sclerosis has not been sufficiently investigated, it is known to be associated with endothelial cell damage, immune system disorders, and excessive extracellular matrix synthesis $[18,19]$. However, the underlying physiological mechanisms of abnormal vascular reactivity, namely, the functional vasoconstriction and structural intimal proliferation and obstruction, are not yet known
[20]. In comparison, HAVS is caused when vibrations stimulate the sympathetic nerves to induce vasoconstriction, and the recurring vasoconstriction eventually leads to the thickening of the arterial wall, causing circulation problems in the hands [11]. As a result, the difference in capillary microscopic findings can be understood as a difference in the pathophysiological mechanism between the two diseases. However, more studies must be conducted to precisely investigate the difference in capillary microscopic findings. Furthermore, through such studies, the pathological parameters in the capillary microscopic findings of HAVS should be determined.

In primary Raynaud's phenomenon, the pattern of the nailfold capillaries remains normal, whereas in systemic sclerosis, a secondary Raynaud's phenomenon, a characteristic pattern of nailfold capillaries is exhibited. Such characteristic patterns include a decrease in capillary density, avascularity, a giant capillary, and hemorrhages $[4,12]$. However, characteristic nailfold capillary microscopic patterns associated with HAVS have not yet been identified. Sakaguchi et al. [16] have classified the capillary loop in HAVS patients according to the number of crossings (Kusumoto's classification system). Compared with the control group, the HAVS group has been shown to exhibit a greater number of crossings in the capillary loop. The present study is meaningful in that the application of the capillary microscopic parameters confirmed the number of crossing capillaries and crossing count, which were greater in the exposed group than in the control group significantly. In addition, although capillary microscopy cannot yet be used alone as a diagnostic tool for HAVS, the characteristic capillary morphological pattern (crossing capillary number and crossing count) described in the study can distinguish patients with HAVS from individuals 
Table 3 Descriptive factors of the observed capillaries (non-VWF vs. VWF)

\begin{tabular}{|c|c|c|c|c|c|}
\hline \multicolumn{2}{|c|}{ Microscopic findings } & \multirow{2}{*}{$\begin{array}{l}\text { Non-VWF }(\mathbf{n}=\mathbf{4 4}) \\
15.14 \pm 3.70\end{array}$} & \multirow{2}{*}{$\begin{array}{l}\text { VWF }(\mathbf{n}=\mathbf{1 8}) \\
15.61 \pm 4.02\end{array}$} & \multirow{2}{*}{$\frac{p \text { value* }}{0.663}$} & \multirow{2}{*}{$\begin{array}{l}\text { Adjusted } \boldsymbol{p} \text { value } \\
0.591\end{array}$} \\
\hline & Afferent 1 & & & & \\
\hline & Afferent 2 & $14.59 \pm 3.59$ & $14.39 \pm 4.01$ & 0.847 & 0.819 \\
\hline \multirow[t]{5}{*}{ Afferent } & Afferent 3 & $13.65 \pm 3.99$ & $13.67 \pm 2.75$ & 0.990 & 0.625 \\
\hline & Afferent mean & $14.46 \pm 3.09$ & $14.55 \pm 3.00$ & 0.915 & 0.952 \\
\hline & Afferent maximum & $16.77 \pm 3.80$ & $16.80 \pm 4.12$ & 0.977 & 0.973 \\
\hline & Top 1 & $17.41 \pm 4.86$ & $19.60 \pm 5.14$ & 0.119 & 0.891 \\
\hline & Top 2 & $17.42 \pm 4.28$ & $17.58 \pm 3.31$ & 0.888 & 0.992 \\
\hline \multirow[t]{5}{*}{ Top } & Top 3 & $16.25 \pm 4.76$ & $16.36 \pm 4.71$ & 0.934 & 0.909 \\
\hline & Top mean & $17.03 \pm 3.75$ & $17.85 \pm 3.32$ & 0.424 & 0.982 \\
\hline & Top maximum & $19.71 \pm 5.07$ & $21.00 \pm 4.84$ & 0.363 & 0.875 \\
\hline & Venous 1 & $20.43 \pm 6.73$ & $19.63 \pm 4.94$ & 0.650 & 0.478 \\
\hline & Venous 2 & $18.14 \pm 3.95$ & $17.40 \pm 5.21$ & 0.545 & 0.505 \\
\hline \multirow[t]{5}{*}{ Venous } & Venous 3 & $17.65 \pm 5.67$ & $17.51 \pm 4.16$ & 0.924 & 0.383 \\
\hline & Venous mean & $18.74 \pm 4.60$ & $18.18 \pm 4.29$ & 0.659 & 0.379 \\
\hline & Venous maximum & $21.81 \pm 6.42$ & $20.59 \pm 4.85$ & 0.471 & 0.293 \\
\hline & Total width 1 & $52.48 \pm 13.26$ & $50.41 \pm 12.02$ & 0.569 & 0.623 \\
\hline & Total width 2 & $48.11 \pm 9.64$ & $49.89 \pm 13.67$ & 0.621 & 0.308 \\
\hline \multirow[t]{5}{*}{ Total width } & Total width 3 & $47.65 \pm 13.42$ & $45.39 \pm 8.09$ & 0.419 & 0.356 \\
\hline & Total width mean & $49.41 \pm 10.37$ & $48.56 \pm 9.46$ & 0.764 & 0.345 \\
\hline & Total width maximum & $56.28 \pm 13.21$ & $55.69 \pm 12.36$ & 0.872 & 0.355 \\
\hline & Length 1 & $341.32 \pm 122.35$ & $392.45 \pm 149.93$ & 0.167 & 0.996 \\
\hline & Length 2 & $321.50 \pm 129.00$ & $384.07 \pm 176.19$ & 0.126 & 0.256 \\
\hline \multirow[t]{3}{*}{ Length } & Length 3 & $334.75 \pm 129.55$ & $343.87 \pm 173.84$ & 0.821 & 0.116 \\
\hline & Length mean & $332.52 \pm 112.75$ & $373.46 \pm 152.51$ & 0.248 & 0.848 \\
\hline & Length maximum & $389.61 \pm 138.84$ & $445.69 \pm 151.15$ & 0.164 & 0.587 \\
\hline
\end{tabular}

Unit: $\mu \mathrm{m}$.

${ }^{*} t$ test.

${ }^{+}$Adjusted for age, smoking status, and diseases using analysis of covariance.

Abbreviations: Non-VWF non- vibration-induced white finger, VWF vibration-induced white finger.

Table 4 Specific counts of morphological findings (non-VWF vs. VWF)

\begin{tabular}{llllll}
\hline Microscopic findings & & Non-VWF $(\mathbf{n}=\mathbf{4 4})$ & VWF $(\mathbf{n}=\mathbf{1 8})$ & $\boldsymbol{p}$ value* & Adjusted $\boldsymbol{p}$ value $^{\boldsymbol{*}}$ \\
\hline \multirow{3}{*}{ Crossing count } & Crossing 1 & $1.66 \pm 0.94$ & $2.33 \pm 0.69$ & 0.008 & 0.058 \\
& Crossing 2 & $1.14 \pm 0.88$ & $2.00 \pm 0.84$ & 0.001 & 0.376 \\
& Crossing 3 & $0.77 \pm 0.80$ & $1.44 \pm 0.78$ & 0.004 & 0.378 \\
& Total crossing & $3.57 \pm 2.35$ & $5.78 \pm 2.02$ & 0.001 & 0.169 \\
Branching count & Branching 1 & $0.59 \pm 0.69$ & $0.22 \pm 0.55$ & 0.032 & 0.280 \\
& Branching 2 & $0.23 \pm 0.42$ & $0.06 \pm 0.24$ & 0.048 & 0.187 \\
& Branching 3 & $0.16 \pm 0.37$ & $0.00 \pm 0.00$ & 0.007 & 0.168 \\
\hline
\end{tabular}

Unit: number.

${ }^{*} t$ test.

${ }^{\dagger}$ Adjusted for age, smoking status, and diseases using analysis of covariance.

Abbreviations: Non-VWF non- vibration-induced white finger, VWF vibration-induced white finger. 
without HAVS. Therefore, capillary microscopy has the potential as a diagnostic indicator of HAVS. Through such discoveries, meaningful data for differential diagnosis of Raynaud's phenomenon can also be provided.

The present study is distinct from the previous studies because we included new parameters that were previously not studied, in particular nailfold capillary microscopy for HAVS. Based on the qualitative factor of capillary morphology and the quantitative factor of capillary dimensions, the respective parameters were quantitatively compared contingent to the presence of HAVS.

However, the present study had several limitations. First, we could not match the ages of the exposed group with those of the control group, and the exposed group was significantly older than the control group. Although age has been shown to only slightly influence blood flow compared with vibration stimulation [16], the influence of age on vibration stimulation cannot be ignored. Second, both the exposed and control groups were comprised primarily of current smokers. Considering the decrease in cutaneous microcirculation due to smoking [21], not excluding subjects with a history of smoking is another limitation of the study. Third, only one examiner performed the nailfold capillary microscopic measurements; therefore, errors in the capillary morphological and dimensional measurements may have occurred, resulting in problems with the reliability of our study. Fourth, the diagnosis of HAVS was only dependent on the Stockholm Workshop Scale, which might have led to errors in diagnosis. The Stockholm stages are difficult to clearly differentiate; therefore, the use of a non-objective diagnostic tool as a stand-alone diagnostic method may also be a limitation. Fifth, the diagnostic utility of capillary microscopy should be evaluated in terms of its sensitivity and specificity and in comparison with those of conventional diagnostic methods. Last, the number of study subjects, especially in the exposed group, was small, thus restricting the power of the analysis to determine a correlation between the capillary microscopic parameters and HAVS.

\section{Conclusions}

In conclusion, HAVS research using capillary microscopy has not been thoroughly studied in South Korea; thus, large-scale studies are needed to confirm the effectiveness of capillary microscopy as a diagnostic tool. In particular, the present study only confirmed the presence of abnormal findings in the exposed and control groups through capillary microscopy. However, the ratio of normal to abnormal capillaries may be helpful and the differences between normal and abnormal capillaries should be compared. Moreover, serological testing using the serum markers soluble thrombomodulin (sTM) or soluble intercellular adhesion molecule-1 (sICAM-1) $[2,22,23]$ as well as prospective studies on a 3- or 6-month cycle can aid in research efforts [6]. Furthermore, capillary microscopic findings differentiated by the Stockholm vascular stage should be analyzed to investigate changes in capillary microscopic parameters with increased stage. However, capillary morphology, dimensions, and specific counting (crossing and branching) did not show a consistent trend according to the increase in Stockholm vascular stage (stages 0-3), and there were no significant differences among stages $0-3$ (data not shown). However, the quantitative and qualitative parametric changes due to stage increases should be analyzed in a future, large-scale study.

\section{Competing interests}

The authors declare that they have no competing interests.

\section{Authors' contributions}

CBL: The first author of this article. He designed the study, collected and interpreted the data, and drafted the manuscript. JHS: He suggested the study design, interpreted the data, and revised the manuscript. CSS: He interpreted the data, revised the manuscript. JHP: He collected the data. CIY: He interpreted the data, revised the manuscript. JSO: He suggested the study design, interpreted the data. HL: He suggested the study design, interpreted the data, and revised the manuscript. All authors read and approved the final manuscript.

\section{Acknowledgments}

This work was supported by Ulsan University Hospital Education \& Research Assistant Manager.

\section{Author details}

${ }^{1}$ Department of Occupational and Environmental Medicine, Ulsan University Hospital, University of Ulsan, College of Medicine, Ulsan, Korea. ${ }^{2}$ Department of Internal Medicine, Ulsan University Hospital, University of Ulsan, College of Medicine, Ulsan, Korea.

Received: 24 December 2013 Accepted: 27 June 2014

Published online: 10 October 2014

\section{References}

1. Harada N, Mahbub M: Diagnosis of vascular injuries caused by handtransmitted vibration. Int Arch Occup Environ Health 2008, 81:507-518.

2. Heaver C, Goonetilleke K, Ferguson H, Shiralkar S: Hand-arm vibration syndrome: a common occupational hazard in industrialized countries. J Hand Surg (European Volume) 2011, 36:354-363.

3. Harada N, Takahashi S, Shirono S, Fujimura H, Morita H, Inagaki J: Occupational exposure limit for hand-arm vibration of the Japan Society for Occupational Health. In Proceedings of the Ninth International Conference on Hand-Arm Vibration INRS. ; 2001:90-95.

4. Mahbub M, Harada N: Review of different quantification methods for the diagnosis of digital vascular abnormalities in hand-arm vibration syndrome. J Occup Health 2011, 53:241-249.

5. Jung P, Trautinger F: Capillary microscopy of toes. J Dtsch Dermatol Ges 2013, 11:855-866.

6. Cutolo M, Sulli A, Smith V: How to perform and interpret capillary microscopy. Best Pract Res Clin Rheumatol 2013, 27:237-248.

7. Koenig M, Joyal F, Fritzler MJ, Roussin A, Abrahamowicz M, Boire G, Goulet JR, Rich E, Grodzicky T, Raymond Y, Senecal JL: Autoantibodies and microvascular damage are independent predictive factors for the progression of Raynaud's phenomenon to systemic sclerosis: a twenty-year prospective study of 586 patients, with validation of proposed criteria for early systemic sclerosis. Arthritis Rheum 2008, 58:3902-3912.

8. Kaji H, Bossnev W, Honma H, Saito K, Tsutsui T, Matsuoka M: Capillary microscopy of finger nailfold as a tool for studying peripheral circulation disorders in hand-arm vibration syndrome. Occupations 2004, 19:17.13.

9. Littleford R, Khan F, Hindley M, Ho M, Belch J: Microvascular abnormalities in patients with vibration white finger. QJM 1997, 90:525-529.

10. Harada N, Iwamoto M, Laskar MS, Hirosawa I, Nakamoto M, Shirono S, Wakui T: Effects of room temperature, seasonal condition and food intake on finger 
skin temperature during cold exposure test for diagnosing

hand-arm vibration syndrome. Ind Health 1998, 36:166.

11. Kim Y: Evaluation of finger skin temperature by cold provocation test for diagnosis of hand-arm vibration syndrome. Ann Occup Environ Med 2002, 14:124-133.

12. Kabasakal Y, Elvins DM, Ring EF, McHugh NJ: Quantitative nailfold capillary microscopy findings in a population with connective tissue disease and in normal healthy controls. Ann Rheum Dis 1996, 55:507-512.

13. Andrade LEC, Gabriel A Jr, Assad RL, Ferrari AJL, Atra E: Panoramic nailfold capillaroscopy: a new reading method and normal range. Semin Arthritis Rheum 1990, 20:21-31.

14. Ingegnoli F, Gualtierotti R, Lubatti C, Zahalkova L, Meani L, Boracchi P, Zeni S, Fantini F: Feasibility of different capillaroscopic measures for identifying nailfold microvascular alterations. Semin Arthritis Rheum 2009, 38:289-295.

15. Wasik JS, Simon RW, Meier T, Steinmann B, Amann-Vesti BR: Nailfold capillary microscopy: Specific features in Fabry disease. Clin Hemorheol Microcirc 2009, 42:99-106.

16. Sakaguchi S, Miyai N, Takemura S, Fukumoto J, Tomura T, Shiozaki M, Kurasawa S, Yokoi K, Terada K, Yoshimasu K, Miyashita K: Morphologic classification of nailfold capillary microscopy in workers exposed to hand-arm vibration. Ind Health 2011, 49:614-618.

17. Varga J, Denton CP, Wigley FM: Scleroderma: From Pathogenesis to Comprehensive Management. Springer; 2011.

18. Jimenez SA, Derk CT: Following the molecular pathways toward an understanding of the pathogenesis of systemic sclerosis. Ann Intern Med 2004, 140:37-50

19. Svegliati Baroni S, Santillo M, Bevilacqua F, Luchetti M, Spadoni T, Mancini M, Fraticelli P, Sambo P, Funaro A, Kazlauskas A: Stimulatory autoantibodies to the PDGF receptor in systemic sclerosis. New Engl J Med 2006, 354:2667-2676.

20. Korn J, Mayes M, Matucci Cerinic M, Rainisio M, Pope J, Hachulla E, Rich E, Carpentier P, Molitor J, Seibold J: Digital ulcers in systemic sclerosis: prevention by treatment with bosentan, an oral endothelin receptor antagonist. Arthritis Rheum 2004, 50:3985-3993.

21. Monfrecola G, Riccio G, Savarese C, Posteraro G, Procaccini E: The acute effect of smoking on cutaneous microcirculation blood flow in habitual smokers and nonsmokers. Dermatology 1998, 197:115-118.

22. Kao DS, Yan J-G, Zhang L-L, Kaplan RE, Riley DA, Matloub HS: Serological tests for diagnosis and staging of hand-arm vibration syndrome (HAVS). Hand 2008, 3:129-134

23. Kennedy G, Khan F, McLaren M, Belch J: Endothelial activation and response in patients with hand arm vibration syndrome. Eur J Clin Invest 1999, 29:577-581.

\section{Submit your next manuscript to BioMed Central and take full advantage of:}

- Convenient online submission

- Thorough peer review

- No space constraints or color figure charges

- Immediate publication on acceptance

- Inclusion in PubMed, CAS, Scopus and Google Scholar

- Research which is freely available for redistribution 